\title{
Evaluación de competencias genéricas en el ámbito universitario a través de entornos virtuales: Una revisión narrativa
}

\author{
Soft Skills assessment through virtual environments in the university sector: A narrative review
}

\author{
Ruiz-Morales, Yovanni ${ }^{(1)}$; García-García, Mercedes $^{(2)}$; Biencinto-López, Chantal ${ }^{(2)}$ \& \\ Carpintero, Elvira ${ }^{(2)}$ \\ (1) Universidad Nacional Experimental del Táchira (Venezuela). (2) Universidad Complutense de Madrid (España)
}

\begin{abstract}
Resumen
El artículo expone una revisión narrativa al estado de la cuestión sobre la enseñanza y evaluación de competencias genéricas a través de Entornos Virtuales (EV) en el ámbito universitario, a partir de la consulta en revistas científicas en formato electrónico e impreso publicadas entre el año 2000 y 2014, así como también de los proyectos de investigación centrados en el desarrollo de competencias genéricas a través de EV. Sintetiza las aportaciones teóricas y empíricas, como una forma de aportar un mayor conocimiento a una línea iniciada hace escasamente una década. Las competencias genéricas, relacionadas con la formación integral del estudiante, al conjugarse con las específicas permiten un mejor desempeño en los ámbitos personal, académico, social y organizacional. Esta premisa supone la necesidad de plantearse el desarrollo de nuevas metodologías para la enseñanza, aprendizaje y evaluación de las competencias genéricas. Entre los resultados se destaca el valor de las competencias genéricas en la formación de los estudiantes universitarios, así como también el desarrollo de proyectos innovadores centrados en la aportación de procedimientos de enseñanza y evaluación viables y eficaces para el logro de competencias genéricas en EV.
\end{abstract}

Palabras clave: Competencias genéricas, enseñanza, aprendizaje, evaluación, entornos virtuales, educación superior.

\begin{abstract}
The paper presents a narrative on the state of the question about the teaching and assessment of generic soft skills through Virtual Environments (VEs) in universities, based on consultation of scientific journals in electronic and printed format published between 2000 and 2014, as well as research projects focused on the development of generic skills through VEs. The paper summarises the theoretical and empirical contributions as a way of providing a greater insight into a line of research that began barely a decade ago. Soft skills related to student training, when combined with specific skills, enable better performance in personal, academic, social and organisational settings. This premise implies the need to consider the development of new methodologies for teaching, learning and assessment of soft skills. Results include the value of soft skills in training university students, as well as the development of innovative projects focused on the provision of teaching and assessment procedures that are feasible and effective for the achievement of soft skills assessment in VEs.
\end{abstract}

Fecha de recepción 2015 Octubre 21

Fecha de aprobación 2017 Enero 20

Fecha de publicación 2017 Enero 20

Reception Date 2015 October 21

Approval Date 2017 January 20

Publication Date: 2017 January 20

Keywords: Soft skills, teaching, learning, assessment, virtual environments, higher education.

La Convergencia hacia el Espacio Europeo de Educación Superior (EEES) plantea a la Universidad una reformulación de las metodologías docentes, orientadas hacia un enfoque de competencias y el diseño de la evaluación formativa durante el proceso de enseñanza y aprendizaje. La clave está en que la formación universitaria cambie su centro de

\section{Autor de contacto / Corresponding author}

Yovanni Ruiz Morales. Universidad Nacional Experimental de Táchira (UNET) Venezuela. 
atención desde los procesos de enseñanza impartidos por el profesorado a los procesos de aprendizaje desarrollados por los estudiantes.

Esta nueva conceptualización resalta los aspectos pedagógicos, e incide directamente en los organizativos y estructurales del currículo. La evaluación es una parte fundamental en este modelo, sobre todo, cuando se apoya en las posibilidades que ofrecen las Tecnologías de la Información y la Comunicación (TIC) en el ámbito educativo actual, concretamente los Entornos Virtuales (EV) para proporcionar feedback informativo e inmediato a cada estudiante individualmente durante el proceso educativo.

De acuerdo con Sangrá (2001) y Peñalosa (2010) los EV facilitan flexibilidad, personalización colaboración e interactividad. Flexibilidad para dar respuesta a las necesidades de aprendizaje de los estudiantes; personalización para ajustar el modelo educativo a su perfil diferencial, haciendo más abierto y amplio el plan de trabajo de las asignaturas así como el acceso a las fuentes de información; colaboración, posibilitando el trabajo en equipo de los estudiantes y la focalización en el logro de metas comunes; e interactividad a la hora de intercambiar datos, procesar información relevante, obtener y ofrecer retroalimentación, ajustar la instrucción al aprendizaje y construir conocimiento. En definitiva, los EV pueden ser más flexibles que el entorno presencial para la implementación de estrategias pedagógicas, ofreciendo niveles de interacción y permitiendo la reutilización de recursos de aprendizaje y la comunicación con variadas aplicaciones informáticas (Sancho Thomas, 2009).

El estudio de las competencias es un tema actual y relevante. En la última década ha generado un amplio número de trabajos teóricos y empíricos (Bolívar, 2008; Climent Bonilla, 2010; Delgado, Borge, García, Oliver \& Salomón, 2005; Díaz Barriga, 2006; Guzmán \& Irigoin, 2000; Mertens, 1997; Vargas, Casanova \& Montanaro, 2001). Parte de los primeros trabajos se centraron en aproximarse a la compresión del concepto de competencia y de su tipología. En este aspecto, González y Wagenaar (2003) desde una visión integradora de las competencias, identifican dos tipos fundamentales: específicas $\mathrm{y}$ genéricas. Las competencias específicas se refieren a una profesión determinada y se adscriben a la disciplina concreta. Estas han sido, precisamente, donde la mayoría de trabajos teóricos (Attewell, 2009; Barberá, 1999; Bolívar, 2008; Bunk, 1994; Castro, 2011; Díaz Barriga, 2006; Escudero, 2008, 2009; García, Andrada, Martel \& Dávila, 2003) se han centrado, intentando clarificar, principalmente, su conceptualización.

Las competencias genéricas, también denominadas transversales, integran lo cognitivo y lo motivacional. Son comunes a todas las profesiones y están constituidas por los siguientes tipos de competencias: a) instrumentales, comprenden las capacidades de comunicación, análisis, síntesis, organización y planificación, así como también de gestión e información, entre otras; b) personales, incluyen las capacidades para el trabajo en equipo, habilidades para el manejo de las relaciones interpersonales y el compromiso ético, entre otras; y c) sistémicas, comprenden la creatividad, el liderazgo, el aprendizaje autónomo y la adaptación a nuevas situaciones, entre otras. A pesar de su reconocida importancia para la formación personal y profesional de los estudiantes (Guitert, Romeu \& Pérez, 2007; Martínez, 2008; Palmer, Montaño \& Palou, 2009) las competencias genéricas han sido objeto de una atención secundaria, sobre todo a la hora de abordar su desarrollo en los centros de Educación Superior.

Este artículo se centra en revisar las fuentes teóricas y empíricas sobre los procedimientos de enseñanza y evaluación de las competencias genéricas en el ámbito universitario en entornos virtuales, partiendo de la delimitación conceptual y tipología realizada por diferentes autores. El objetivo es plantear el estado de la cuestión y delimitar el punto de partida por dónde se debe de avanzar en el conocimiento e investigación de esta línea. 


\section{Las competencias en el ámbito curricular universitario: delimitación conceptual}

En los últimos años se ha escrito mucho sobre la conceptualización de las competencias con el intento de ofrecer una base teórica sólida a las distintas propuestas e innovaciones planteadas desde este enfoque. A pesar de la intensa producción especializada, sigue sin haber una definición única ni universal, más bien son varias las acepciones del concepto dependiendo del contexto donde se enmarquen. Además, se pone en evidencia que aún existe una confusión e imprecisión conceptual y terminológica sobre el constructo competencia (Attewel, 2009; Bunk, 1994; Carabaña, 2011; Delors, 1996; De La Orden, 2011a; De La Orden, 2011b; Díaz, 2006; Escudero, 2008; Le Boterf, 2001; Perrenoud, 2004; Rodríguez, Hernández \& Díaz, 2007; Sáez, 2009; Tejada, 2005; Zabalza, 2001).

De la revisión bibliográfica realizada sobre la conceptuación de las competencias en el ámbito educativo hay consenso en afirmar que comparten dos significados de igual importancia: a) desde el plano teórico, es una estructura cognitiva que permite el desarrollo de conductas específicas; y b) desde el plano operativo, interrelaciona habilidades para accionar conocimientos, actitudes y pensamientos en situaciones complejas (Sáez, 2009). Más concretamente:

- Es un constructo molar, un complejo entramado de conocimientos, habilidades y actitudes que los individuos necesitan para desarrollar algún tipo de actividad en una situación concreta, o contexto específico, donde se ponen en evidencia las actuaciones derivadas de la competencia puesta en ejecución (Zabalza, 2001).

- Constituye un conjunto de capacidades reales para alcanzar objetivos personales, grupales, laborales u organizacionales, mediante la movilización de aptitudes, habilidades y destrezas (Vargas, 2006).

- Supone una descripción de los aprendizajes de los estudiantes que incluye recursos cognitivos, personales, sociales y valores, que tienen que ser aplicados e integrados adecuadamente a situaciones propias del contexto, teniendo en cuenta criterios de tipo social y éticos (Escudero, 2008).

- Constituye un saber hacer complejo, adaptativo y personal, es decir, no se aplica de forma mecánica si no reflexiva. Tiene carácter integrador y se adecua a diversos contextos y situaciones, por lo que abarca conocimientos, habilidades, emociones, valores y actitudes (Pérez \& Soto, 2009).

- Representa un atributo latente que integra conocimiento, actitud, habilidad y destreza para el desarrollo de una profesión, puesto de trabajo o actuación académica, permitiendo dominar las actuaciones académicas o laborales en el nivel exigido (Ibarra Sáiz \& Rodríguez Gómez, 2010).

- Actuaciones integrales para identificar, interpretar, argumentar y resolver problemas con idoneidad y compromiso ético, movilizando el saber conceptual, procedimental y actitudinal (Tobón, Pimienta \& García, 2010).

- Conjunto de conocimientos, habilidades, procedimientos, actitudes y técnicas, que una persona posee y que son necesarias para resolver problemas de forma autónoma, libre y creativa (Jornet, González, Suárez \& Perales, 2011).

Entonces se podría decir que la posición común de la revisión realizada nos lleva a plantear el constructo competencia en el ámbito curricular universitario, como un entramado de saberes complejos (conocimientos, habilidades y destrezas), que conjugados con actitudes (motivación, interés, disposición, etc.) y valores, permiten actuar con asertividad en diversos contextos para hacer frente o dar solución a situaciones propias de las dimensiones del ser humano (individual, familiar, académico, organizacional y social). Para el desempeño de la competencia es fundamental la adecuación del saber y del saber hacer, así como también la disposición de la persona para querer poner en funcionamiento los comportamientos que conforman a la competencia (Ruíz Morales, 2013). 
Desde esta postura, se enfatizan tres aspectos: a) las competencias se desempeñan de forma articulada para resolver problemas complejos, b) los recursos cognitivos y las actitudes son necesarios para que una persona desarrolle comportamientos adecuados con responsabilidad, y c) el contexto donde se ejecuta la competencia juega un papel importante porque es allí donde se determina el cumplimiento de unos criterios o estándares establecidos.

\section{Las competencias genéricas en la formación universitaria}

Las competencias genéricas son comunes a todas las profesiones $\mathrm{y}$, a su vez, están constituidas por tres tipos de competencias: a) instrumentales, comprenden las capacidades de análisis y síntesis, de organización y planificación, así como también de gestión e información; b) personales, incluyen las capacidades para el trabajo en equipo, habilidades para el manejo de las relaciones interpersonales y el compromiso ético; y c) sistémicas, comprenden la creatividad, el liderazgo, el aprendizaje autónomo y la adaptación a nuevas situaciones, entre otras competencias.

Entre los expertos hay acuerdo sobre la importancia de desarrollar las competencias genéricas en el contexto universitario durante la formación del estudiante porque contribuyen al desempeño efectivo en el ámbito académico pero, sobre todo, en el campo laboral siendo un elemento de adaptación y un motor de aprendizaje, que permite seguir aprendiendo a lo largo de la vida. Las condiciones de desempeño profesional en la actualidad exigen, por una parte, conocimientos y competencias específicas propias del ejercicio de una profesión (Le Deist \& Winterton, 2005) pero, por otra, competencias genéricas que permitan ejercer eficientemente la profesión en contextos diversos, con autonomía, flexibilidad, ética y responsabilidad (Concepción, 2012; González \& González, 2008; McMurtrey, Downey, Zeltmann \& Friedman, 2008; Villa \& Poblete, 2011).
Aunque en el ámbito universitario la mayor preocupación es el desarrollo de competencias específicas de la profesión, no hay duda de que las competencias genéricas destacan en los contextos profesionales actuales, principalmente se demandan competencias interpersonales o socio-comunicativas. Parece necesario, en consecuencia, conectar los contextos formativos y laborales de forma que los titulados universitarios estén equipados con mayores niveles de habilidades sociales $\mathrm{y}$ personales para adaptarse mejor a los contextos profesionales. Este planteamiento representa un reto para la didáctica, es decir, una reflexión y evaluación de los métodos de enseñanza, aprendizaje y evaluación en los sistemas universitarios (Rogmann, 2008; Villa \& Poblete, 2007).

Las competencias genéricas que se resaltan para un buen desempeño profesional suelen ser comunes a diferentes titulaciones. Y, aunque profesorado y estudiantes valoran como importantes las competencias genéricas, difieren en la priorización. Así, por ejemplo, mientras que los estudiantes y docentes de la Diplomatura de Ciencias Empresariales de la Universidad de Girona ordenan las competencias genéricas para su perfil formativo en el orden siguiente: responsabilidad, autoformación, planificación, comunicación, relaciones interpersonales, creatividad y liderazgo (Corominas, 2001). El profesorado, por su parte, prioriza como importante en el perfil de formación de los estudiantes las competencias genéricas referidas a la toma de decisiones, solución de problemas, comunicación, gestión de la información, trabajo en equipo, relaciones interpersonales, disposición hacia la calidad y liderazgo (Corominas, Tesouro, Capell, Teixidó, Pélach \& Cortada, 2006).

Este planteamiento destaca que si se pretende que los estudiantes se incorporen mejor preparados al mundo laboral hay que promover espacios de formación en la universidad, antesala de la experiencia profesional superior, que atiendan la formación del conocimiento en un área particular, pero también las habilidades y 
actitudes que permiten llevar a la acción dicho conocimiento en contextos diversos y reales. Asimismo, destaca la responsabilidad que los docentes universitarios tienen respecto al diseño e implementación de estrategias metodológicas que ofrezcan a los estudiantes escenarios formativos orientados al aprendizaje y evaluación de competencias genéricas significativas para su profesión, de forma que contribuyan a la formación integral de un profesional con capacidades para ubicarse adecuadamente en diversos contextos personales, sociales y organizacionales.

\section{Entornos virtuales para el desarrollo de competencias genéricas: investigación previa en España}

Aunque existe acuerdo en afirmar la importancia de las diferentes competencias genéricas para el desarrollo eficiente de los estudiantes y profesionales, ¿cómo se ha abordado hasta ahora la enseñanza y la evaluación de estas competencias en los estudios superiores?

Son varias las experiencias que ponen de manifiesto que se han diseñado y probado diversos procedimientos para el desarrollo y evaluación de competencia genéricas a través de EV, destacando dos evidencias: la primera es la tendencia al uso del e-portafolio para el seguimiento del proceso de aprendizaje y evaluación del estudiante y, la segunda, es la exploración y desarrollo de los recursos electrónicos como apoyo a una evaluación dinámica, continuada, auténtica, de reflexión y diálogo entre sus actores (estudiantes y docentes).

Así, uno de los primeros planteamientos es el uso del portafolio como metodología de evaluación y aprendizaje apoyada en las tecnologías. El trabajo de Barragán (2005) avala el uso del portafolio como una experiencia de evaluación satisfactoria que permite alcanzar las competencias creatividad, compromiso ético, habilidades comunicativas y desarrollo de habilidades para la resolución de problemas.

Además, Bernal, Arráiz, Sabirón, Bueno, Cortés y Escudero (2006), basándose en referentes teóricos del constructivismo social y de la evaluación auténtica, presentan el eportafolio etnográfico como una herramienta versátil para la evaluación de competencias. Esta herramienta resulta clave tanto en el proceso de acompañamiento como para el desarrollo de las competencias pensamiento dialéctico, comportamiento dialógico, estrategias de afrontamiento y autodeterminación.

Capllonch y Castejón (2007) desarrollan una experiencia colaborativa en red, concretamente un e-diario interactivo para la formación inicial de estudiantes de Magisterio de Educación Física de la Universidad Complutense de Madrid y de la Universidad de Barcelona. Los resultados de este trabajo confirman la utilidad de esta herramienta para la autoevaluación, así como también para compartir ideas, reflexiones y conocimientos entre pares.

Por su parte, Guitert, Romeu y Pérez (2007) se plantean el desarrollo de competencias genéricas en estudiantes universitarios, mediante EV. La experiencia reporta resultados favorables hacia la adquisición de las competencias relacionadas con la sociedad de la información, especialmente, búsqueda, análisis, síntesis y gestión de la información digital, así como su procesamiento, organización y presentación. De igual forma, los estudiantes mejoran el aprendizaje de las competencias trabajo en equipo, planificación y organización del trabajo individual y grupal.

Los trabajos de Guasch, Guárdia y Barberá (2009), Barragán, García, Buzón, Rebollo y Vega (2009), Barberá, Gewerc y Rodríguez (2009), Cabero, López y Llorente (2012), Aguaded, López y Jaén (2013), Valverde Berrocoso y Ciudad Gómez (2014) ofrecen un panorama sobre la innovación en la evaluación de los aprendizajes en el ámbito de la Educación Superior, al desarrollar propuestas con portafolios electrónicos como instrumentos didácticos para valorar los aprendizajes y dejan abiertas nuevas líneas de trabajo en el campo de la evaluación de las competencias genéricas. Estos trabajos muestran que el e-portafolio es una 
herramienta pedagógica que permite evaluar competencias y aporta beneficios tanto al estudiante, como al docente, en el sentido de ofrecer oportunidades para: a) registrar evidencias de desempeño, b) retroalimentar a lo largo del proceso de aprendizaje a través del seguimiento de las evidencias de desempeño, c) promover el diálogo, la reflexión y la colaboración entre pares; d) interactuar entre estudiantes, docentes, competencias y contextos; e) evaluar, específicamente, competencias genéricas, tales como: planificación, toma de decisiones y autonomía en el proceso de aprendizaje; y f) evaluar continuamente para la mejora y la autorregulación del aprendizaje.

Ibarra Sáiz (2008) dirige el Proyecto Evalcomix, centrado en la evaluación de competencias en un contexto de aprendizaje blearning en los másteres oficiales del área de las Ciencias Sociales y Jurídicas, impartidos en nueve Universidades Españolas. Como resultado de este trabajo se diseña un portal de Internet que ofrece al profesorado universitario la posibilidad de crear y compartir procedimientos, herramientas e instrumentos prácticos, criterios e indicadores, susceptibles de ser utilizados en entornos de e-Learning. Su aportación es fundamental puesto que ofrece una herramienta metodológica útil para el seguimiento y evaluación de competencias de los estudiantes de posgrado del área de las Ciencias Sociales.

Otros trabajos (Cebrián de la Serna \& Bergman Stockholm, 2014; Lázaro y Torres, Ruíz Palomeque \& González González, 2009; Martín, León \& García, 2014; Moreno-Crespo, López Noguero \& Cruz Díaz, 2014; Olmos \& Rodríguez, 2008) ponen en evidencia los beneficios que aportan las tecnologías al proceso de evaluación. Concretamente, los estudiantes pueden valorar sus conocimientos a través de la autoevaluación, la retroalimentación inmediata y con la posibilidad de conocer los niveles de logro alcanzados. Los resultados obtenidos confirman la autonomía que se le concede al estudiante con este tipo de evaluaciones formativas en la red.
El Proyecto Evalhida (Rodríguez, 2008) plantea el análisis de la utilización de herramientas de interacción dialógica asíncrona (hida) para evaluar algunas competencias genéricas de los estudiantes en la realización activa y participativa de una tarea centrada en los Blogs, Foros y Wikis. Entre las conclusiones destacan las siguientes: a) la evaluación de competencias con una herramienta de interacción dialógica asíncrona puede realizarse aplicando rúbricas, listas de control y escalas de valoración; y b) las hida actuales no facilitan la aplicación de procedimientos de evaluación, puesto que no reflejan cómo las contribuciones a un wiki o blog constituyen evidencias de las competencias alcanzadas.

Herradón, Blanco, Pérez y Sánchez (2009) realizan un trabajo centrado en el aprendizaje y evaluación de competencias genéricas en la Universidad Politécnica de Madrid. El procedimiento de formación utilizado fue blearning, mediante la plataforma Moodle. En concreto, abordan las competencias búsqueda y selección de información, defensa de argumentos, trabajo en equipo y manejo de las TIC. Esta experiencia confirma la importancia de utilizar rúbricas para evaluar competencias ya que permiten establecer con claridad los criterios de evaluación y que estos sean conocidos por los estudiantes. Cabe destacar de esta experiencia la dificultad para la evaluación de la competencia trabajo en equipo, debido a la actitud de resistencia mostrada por los estudiantes hacia la asunción de responsabilidades en la toma de decisiones y consecución de objetivos comunes durante la realización del proyecto.

Con objeto de ofrecer situaciones motivadoras y estimulantes para los estudiantes, Sancho Thomas (2009) diseña un sistema de aprendizaje virtual colaborativo escenificado a través de un juego de rol multijugador, en la Licenciatura en Ciencias Físicas de la Universidad Complutense de Madrid. Los resultados mostraron una reducción de la tasa de abandono entre los estudiantes y la multiplicación por cinco del número de interacciones a través del campus 
virtual, frente a un escenario tradicional. Estos resultados coinciden con los planteamientos de Cabero y Llorente (2007), Gros, García y Lara (2009), Maldonado, Leal y Montenegro (2009) y Peñalosa (2010) quienes muestran evidencias sobre las prestaciones que ofrece la tecnología para la interacción didáctica y el trabajo en equipo entre estudiantes.

Apoyado en los resultados de Sancho Thomas (2009) y los proyectos del grupo de investigación Evaluación en Contextos Formativos (Evalfor) se desarrolla el proyecto Evalsoft, un portal de internet que permite la creación, almacenamiento y uso de procedimientos e instrumentos de evaluación de cuatro competencias genéricas: compromiso, comunicación, liderazgo y trabajo en equipo (García, 2010). El estudio aporta la orientación pedagógica que se le brinda al docente universitario, cualquiera que sea su área de trabajo, para la evaluación del grado de desarrollo de dichas competencias en los estudiantes.

Del Canto et al. (2010) implementan el campus digital Atenea, el cual permite gestionar las entregas de trabajos realizados por los estudiantes universitarios. Este espacio virtual facilita la realización de la evaluación, el seguimiento y la retroalimentación del aprendizaje de los estudiantes. Concretamente, ofrece al docente la posibilidad de: a) plantear las tareas que deben realizar los estudiantes, b) definir las fechas de entrega, c) gestionar la entrega de trabajos en formato digital, d) retroalimentar cada trabajo, con apreciaciones cualitativas que deben ser incorporadas en sucesivas entregas para su posterior calificación; y e) establecer la comunicación docente-estudiante.

En la línea de involucrar a los estudiantes activamente en los procesos de evaluación utilizando las TIC, Nicol y Macfarlane (2006) y Nicol (2009a; 2009b; 2010) desarrollan el Proyectos PEER (evaluación por revisión entre pares) y REAP (reingeniería en las prácticas de evaluación) que ofrece a los estudiantes la oportunidad de valorar su propio trabajo y el de otros. La implementación de este sistema pone en evidencia que la evaluación entre pares, la retroalimentación y proalimentación facilitan el desarrollo del pensamiento crítico de los estudiantes, así como también la capacidad para supervisar, evaluar y gestionar el aprendizaje. De igual modo se confirma que la evaluación colaborativa es una estrategia plausible cuando se quiere generar nuevas formas de participación e implicación de los estudiantes universitarios como agentes de su aprendizaje.

García (2009), desarrolla el Portal Web "Ecompetentis" para la evaluación de competencias transversales. Esta herramienta permite al profesorado universitario la inclusión, valoración y difusión de actividades pedagógicas orientadas a la formación y evaluación de las siguientes competencias: comunicación oral y escrita, trabajo en equipo, resolución de problemas y uso de idiomas extranjeros. También se ofrecen instrumentos de evaluación y proyectos de innovación e investigación que se pueden contextualizar en asignaturas del ámbito universitario.

En el marco de referencia de la evaluación orientada al aprendizaje (Carless, Joughin \& Liu, 2006), Gómez Ruíz, Rodríguez Gómez e Ibarra Sáiz (2013) plantean la e-Evaluación orientada al aprendizaje (e-EOA) "como un proceso de aprendizaje, mediado por las TIC, que promueve el desarrollo de competencias útiles para el presente académico y el futuro laboral de los estudiantes" (p. 1), desde esta premisa realizan una investigación cuasiexperimental, con diseño pretest-postest con grupos experimentales y de control para comprobar el impacto que la e-EOA tiene en la percepción del nivel competencial de estudiantes universitarios pertenecientes al área de Ciencias Sociales y Jurídicas, los resultados revelan que en los grupos experimentales se mejora el nivel competencial y existen diferencias significativas entre su pretest-postest. Además, se comprueba que las competencias genéricas que más se han mejorado de forma significativa han sido: el aprendizaje autónomo, la resolución de problemas y la creatividad. 
Apoyado principalmente en los resultados del Proyecto Evalcomix (Ibarra Sáiz, 2008) y el Proyecto Evalsoft (García, 2010), Ruíz Morales (2013) presenta un entorno virtual pedagógico, soportado por Moodle y en la modalidad instruccional b-learning, que constituye una metodología para la enseñanza, aprendizaje y evaluación en el ámbito universitario. En concreto, mediante procedimientos electrónicos de autoevaluación, evaluación de pares y evaluación del profesorado muestra que un EV colaborativo, favorece el desarrollo de las competencias genéricas comunicación, innovación, liderazgo, compromiso y, principalmente, el trabajo en equipo.

El estudio realizado por Myers, Blackman, Andersen, Hay y Lee (2014) pone de manifiesto que el trabajo en equipo es una competencia que se puede desarrollar y evaluar apoyándose en las TIC, específicamente con el uso de algunas redes sociales, blogs y facebook, los estudiantes se comunican y colaboran en la realización de trabajos de investigación, acompañados del Feedback que ofrece el docente a través de la mediación tutorial.

De los trabajos revisados, centrados en el desarrollo del aprendizaje-evaluación mediante EV y en los efectos de los EV en la mejora de competencias formativas, destacan cuatro aspectos que pudieran orientar el planteamiento de los nuevos proyectos de investigación sobre el aprendizaje y la evaluación de competencias genéricas:

- El aprendizaje y evaluación de competencias genéricas, mediante tecnología y concretamente a través de EV se ha centrado, hasta la fecha, más frecuentemente en el desarrollo de competencias relacionadas con la sociedad de la información, la planificación del trabajo, la toma de decisiones, la resolución de problemas y el trabajo en equipo. Por el contrario, es escasa la investigación orientada a la enseñanza, aprendizaje y evaluación de la capacidad para comunicarse adecuadamente por escrito, necesaria para el uso adecuado del lenguaje técnico-académico, así como también es insuficiente el desarrollo de habilidades para liderar equipos de trabajo y las capacidades para innovar mediante la generación y experimentación de ideas.

- El e-portafolio se presenta como un instrumento adecuado para la evaluación de competencias genéricas en el ámbito universitario. Sobre todo para la autoevaluación y evaluación de pares, puesto que permite el registro sistemático de evidencias del aprendizaje adquirido y el seguimiento a las producciones de los estudiantes durante el proceso educativo apoyado en tecnología.

- Los estudiantes que se inician en experiencias virtuales colaborativas aumentan su satisfacción, implicación, percepción del aprendizaje conseguido y cómo mejorarlo. Además los docentes también muestran mayor satisfacción con los resultados y los niveles de logro competencial alcanzado por sus estudiantes.

- La tendencia actual es el uso de la evaluación formativa con autoevaluación y evaluación colaborativa, a través de un conjunto de acciones pedagógicasinteractivas y recursos electrónicos que ofrezcan oportunidades didácticas y formativas al estudiante para aprender habilidades sociales a través de su propia evaluación y con apoyo de la tecnología.

De acuerdo con Suárez, Pérez, Boza y García-Valcárcel (2013) este panorama impone cambios profundos en los procesos de integración de las TIC al mundo de la educación, en particular los EV para el aprendizaje y la evaluación. Por un lado, exige modificar el papel y la práctica pedagógica del profesorado, quienes tienen la responsabilidad de planificar, diseñar e implementar intervenciones, desde la perspectiva de la diversidad, centradas en la actividad y participación de los estudiantes utilizando las tecnologías que la sociedad pone a su alcance. Por otro lado, es fundamental que el estudiante desarrolle capacidades que le permitan seguir aprendiendo de manera cada vez más eficaz y 
autónoma, de acuerdo con sus necesidades y objetivos $\mathrm{y}$ en un entorno sociocultural cambiante. Es decir, un estudiante que ya no tiene que ser acumulador o reproductor de conocimiento sino que, sobre todo, debe aprender a adaptarse a realidades cambiantes, ser capaz de trabajar y aportar con sus pares para el logro de objetivos comunes y ser un usuario inteligente de la información, flexible, adaptándose a los cambios a lo largo de la vida.

También se producen cambios en las instituciones educativas cuando se opta por incorporar $\mathrm{EV}$ con la finalidad de formar personas autónomas, eficaces, responsables, críticas, reflexivas, con dominio del lenguaje oral y escrito, que resuelvan situaciones de la vida cotidiana, de un modo eficiente. Así como señalan Martín Sánchez y López Meneses (2012), la cuestión está en propiciar entornos virtuales educativos que posibiliten la interacción y relación educativa sin apenas límites espaciales, geográficos y temporales, que en definitiva inviten a la construcción del conocimiento, la experimentación y la resolución de problemas, a escala individual y grupal.

\section{Conclusiones}

La incorporación de los EV en el currículo universitario $\mathrm{y}$, particularmente, en la enseñanza, aprendizaje y evaluación de diferentes competencias, ha ido creciendo gracias a las facilidades de conectividad, flexibilidad, interacción, autonomía, colaboración y motivación que ofrecen las TIC desde marcos pedagógicos (Iriondo \& Gallego, 2013). Pero, es necesario trascender el uso de los EV como repositorios de información, por otra parte su uso más frecuente, para lo que se requiere una planificación didáctica y rigurosa de la formación en red. Es decir, es fundamental un proceso sistemático que contemple las metas educativas y los objetivos de aprendizaje que deben ser alcanzados por los estudiantes, así como también las tareas, los medios, las herramientas y los recursos destinados para el aprendizaje y la evaluación electrónica.

Una de las tendencias más actuales y con mayor proyección, pero insuficientemente estudiada, se centra en el diseño de acciones orientadas al desarrollo de competencias genéricas mediante procedimientos electrónicos. Esto, como se ha puesto de manifiesto, requiere seguir apostando por grupos docentes que, vinculados con la investigación, den viabilidad a innovaciones en el campo del aprendizaje y evaluación de este tipo de competencias en el ámbito universitario. Parece ser una de las mejores vías para producir conocimiento educativo además de facilitar aportes teóricos, metodológicos y prácticos en esta área de interés para la Educación Superior y de gran importancia para los futuros profesionales que deberán rendir cuentas en la sociedad actual.

Las investigaciones sobre el aprendizaje y la evaluación de competencias genéricas a través de entornos virtuales se han iniciado en la última década por lo que todavía es pronto para tener una teoría consistente ni procedimientos suficientemente viables y eficaces. Sin embargo, la revisión realizada pone de manifiesto que esta línea de investigación está produciendo nuevo conocimiento que gira en torno a tres líneas: 1) la evaluación orientada al aprendizaje, 2) la evaluación de competencias mediante herramientas virtuales y colaborativas, y 3) la formación del profesorado en el desarrollo de competencias para la implementación de la eevaluación en la enseñanza-aprendizaje.

Asimismo, la teoría y la investigación realizada hasta el momento sugiere que un proceso de aprendizaje y evaluación de competencias genéricas del estudiante universitario debe plantearse desde un sistema que se caracterice por: a) ser participativo, reflexivo, y crítico entre profesorado y estudiantes; b) valorar el desempeño en las dimensiones del saber, saber hacer y convivir; c) utilizar metodologías y procedimientos contextualizados; d) descubrir y potenciar las fortalezas y aspectos individuales que se deben 
mejorar, de forma personalizada, así como las oportunidades detectadas durante el proceso aprendizaje; y e) consensuar criterios de evaluación e indicadores de logro conocidos y compartidos con los estudiantes.

Los resultados apuntan que los EV pueden apoyar el aprendizaje de las competencias comunicación, planificación y trabajo en equipo, así como la satisfacción de estudiantes y profesorado que han utilizado este tipo de entornos para el desarrollo de competencias en el ámbito universitario. Sin embargo, no hay todavía evidencias concluyentes respecto a la utilidad ni viabilidad de los EV para el abordaje del aprendizaje y la evaluación de competencias genéricas (Ruíz Morales, 2013). En general, la forma de aprender, enseñar o evaluar la mayoría de las competencias genéricas a través de $\mathrm{EV}$ es un tema que aún está por mejorar, profundizar y desarrollar en el ámbito de la Educación Superior. La investigación en esta línea se encuentra en estadios iniciales y aún está por producir teorías consistentes y procedimientos eficaces.

En definitiva, aunque todavía es necesario preguntarse qué inversión supone el desarrollo y evaluación de competencias genéricas a través de entornos virtuales, sin embargo, los resultados apuntan a que es importante seguir indagando sobre las nuevas formas de enseñanza y aprendizaje en el ámbito universitario, aprovechando la potencialidad de los entornos virtuales para generar y construir conocimiento pedagógico. Queda por definir con certeza los modos y acciones más eficaces, pero parece que el camino ya está iniciado.

\section{Referencias}

Aguaded, J.I.; López, E. \& Jaén, A. (2013). Portafolios electrónicos universitarios para una nueva metodología de enseñanza superior. Desarrollo de un material educativo multimedia. Revista de Universidad y Sociedad del Conocimiento, 10(1). doi: http://doi.org/10.7238/rusc.v10i1.1333

Attewell, P. (2009). ¿Qué es una competencia?. Pedagogía Social Revista Interuniversitaria, 16;
21-44.

doi:

https://doi.org/10.7179/PSRI_2009.16.03

Barberá, E. (1999). Evaluación de la enseñanza, evaluación del aprendizaje. Barcelona: EDEBÉ.

Barberá, E.; Gewerc, A. \& Rodríguez, J.L. (2009). Portafolios electrónicos y educación superior en España: Situación y tendencias. Revista de Educación a Distancia. doi: http://doi.org/10.7238/rusc.v10i1.1333

Barragán, R. (2005). El Portafolio, metodología de evaluación y aprendizaje de cara al nuevo Espacio Europeo de Educación Superior. Una experiencia práctica en la Universidad de Sevilla. Revista Latinoamericana de Tecnología Educativa, 4(1), 121-139.

Barragán, R.; García, R.; Buzón, O.; Rebollo, M.A. \& Vega, L. (2009). E-portafolios en Procesos Blended-learning: Innovaciones de la Evaluación en los Créditos Europeos. Revista de Educación a Distancia, Recuperado de: http://www.um.es/ead/red/M8/us.pdf

Bernal, J.L.; Arráiz, A.; Sabirón, F.; Bueno, C.; Cortés, M.P. \& Escudero, T. (2006). El Portafolio-Etnográfico de evaluación de competencias: alternativa a la evaluación en el Espacio Europeo de Educación Superior. Anuario de Pedagogía, Universidad de Zaragoza, 8, 1134.

Bolívar, A. (2008). El discurso de las competencias en España: Educación Básica y Educación Superior. Revista de Docencia Universitaria, 6(2). Recuperado de: http://www.um.es/ead/Red_U/m2/bolivar.pdf

Bunk, G. P. (1994). La transmisión de competencias en la formación y el perfeccionamiento profesional de la República Federal Alemana. Revista Europea de Formación Profesional, 2, 8-14.

Cabero, J. \& Llorente, M. (2007). La interacción en el aprendizaje en red: uso de herramientas, elementos de análisis y posibilidades educativas. Revista Iberoamericana de Educación a Distancia, 10(2), 97-123. doi: http://doi.org/10.5944/ried.2.10.995

Cabero, J.; López, E. \& Llorente, M. (2012). EPortafolio universitario como instrumento didáctico 2.0 para la reflexión, evaluación e investigación de la práctica educativa en el espacio europeo de educación superior. Revista Virtualidad, Educación y Ciencia, 3(4). 
Recuperado

de:

http://revistas.unc.edu.ar/index.php/vesc/article/v $\underline{\text { iew/1886 }}$

Capllonch, M. \& Castejón, F.J. (2007). La adquisición de competencias genéricas a través de una comunidad de práctica y aprendizaje. En J. L. Rodríguez (coord.), Comunidades virtuales de práctica y de aprendizaje. Revista Electrónica Teoría de la Educación: Educación y Cultura en la Sociedad de la Información, 8(3). Recuperado de:

http://gredos.usal.es/jspui/bitstream/10366/56586 11/TEE2007_V8N3 P168.pdf

Carabaña, J. (2011). Competencias y Universidad, o un desajuste por mutua ignorancia. Bordón, 63(1), 15-31.

Carless, D. Joughin, G. \& Liu, N.F. (2006). How assessment supports learning: learning-oriented assessment in action. Hong Kong University Press. doi: https://doi.org/10.5790/hongkong/978962209 8237.001.0001

Castro, M. (2011). ¿Qué sabemos de la medida de las competencias? Características y problemas psicométricos en la evaluación de competencias. Bordón, 63(1), 109-123.

Cebrián de la Serna, M. \& Bergman Stockholm, Ma. E. (2014). Evaluación formativa con erúbrica: aproximación al estado del arte. REDU. Revista de docencia Universitaria, 12(1), 15-22. Recuperado de: http://redu.net/redu/index.php/REDU/article/view/807

Climent Bonilla, J. (2010). Reflexiones sobre la Educación Basada en Competencias. Revista Complutense de Educación, 21(1), 91-106.

Concepción, Y. (2012). Modalidades de evaluación de competencias genéricas en la formación universitaria. Didac, 60, 15-19.

Corominas, E. (2001). Competencias genéricas en la formación universitaria. Revista de Educación, 325, 299-321.

Corominas, E.; Tesouro, M.; Capell, D.; Teixidó, J.; Pélach, J. \& Cortada, R. (2006). Percepciones del profesorado ante la incorporación de las competencias genéricas en la formación universitaria. Revista de Educación, 341, 301336.

De La Orden, A. (2011a). El problema de las competencias en la educación general. Bordón, 63(1), 47-61.
De La Orden, A. (2011b). Reflexiones en torno a las competencias como objeto de evaluación en el ámbito educativo. Revista Electrónica de Investigación Educativa, 13(2), 1-21. Recuperado de:

http://redie.uabc.mx/index.php/redie/article/view/ $\underline{278}$

Delgado, A.M.; Borge, R.; García, J.; Oliver, R. \& Salomón, L. (2005). Competencias y Diseño de la Evaluación Continua y Final en el Espacio Europeo de Educación Superior. Madrid: Ministerio de Educación y Ciencia. doi: http://doi.org/10.13140/2.1.4874.5928

Delors, J. (1996). La educación encierra un tesoro. Madrid: Editorial Santillana. UNESCO.

Del Canto, P.; Gallego, I.; López, J. M.; Mochón, F.; Mora, J.; Reyes, A. et al. (2010). La evaluación en el contexto del Espacio Europeo de educación Superior. Revista de Educación a Distancia. Recuperado de: http://www.um.es/ead/reddusc/1/valero.pdf

Díaz Barriga, A. (2006). El Enfoque de Competencias en la Educación. ¿Una alternativa o un disfraz de cambio? Perfiles Educativos, 28(111), 7-36.

Escudero, J.M. (2008). Las competencias profesionales y la formación universitaria: posibilidades y riesgos. Revista de Docencia Universitaria, $1 . \quad$ Recuperado de: http://umd.upla.cl/cursos/fmdelbuey/marco_com petencias/competencias profesionales formacion _universitaria.pdf

Escudero, J.M. (2009). Las competencias profesionales y la formación universitaria: posibilidades y riesgos. Pedagogía Social Revista Interuniversitaria, 16, 65-82. doi: https://doi.org/10.7179/PSRI_2009.16.05

García, M., Andrada, J., Martel, M. \& Dávila, N. (2003, 11-12 de septiembre). Una propuesta de evaluación continua para Matemáticas I. Análisis de los resultados. Comunicación presentada en las XI Jornadas de ASEPUMA, Oviedo. Recuperado de: http://www.uv.es/asepuma/XI/33.pdf

García, M. (dir.) (2010). Proyecto Evalsoft: Evaluación de Competencias Interpersonales en Entornos Virtuales de Aprendizaje. Programa de estudios y análisis para la mejora de la calidad de la enseñanza superior y de la actividad del profesorado universitario. Secretaría de estado de 
Universidades. Universidad Complutense de Madrid.

García, M.J. (coord.) (2009). Desarrollo de la herramienta eCompetentis para la evaluación de competencias transversales. Programa de Estudios y Análisis destinados a la mejora de la calidad de la enseñanza superior y de la actividad del profesorado universitario. Madrid: Servicio de Publicaciones de la Universidad Politécnica de Madrid.

Gómez Ruíz, M. Á.; Rodríguez Gómez, G. \& Ibarra Sáiz, Ma.S. (2013). Desarrollo de las competencias básicas de los estudiantes de Educación Superior mediante la e-Evaluación orientada al aprendizaje. Revista Electrónica de Investigación y Evaluación Educativa, 19(1). doi: http://doi.org/10.7203/relieve.19.1.2457

González, V. \& González, R. (2008). Competencias genéricas y formación profesional: un análisis desde la docencia universitaria. Revista Iberoamericana de Educación, 47, 185209.

González, J. \& Wagenaar, R. (eds.) (2003). Tuning Educational Structures in Europe. Informe Final. Fase Uno. Bilbao: Universidad de Deusto.

Guitert, M.; Romeu, T. \& Pérez, M. (2007). Competencias TIC y trabajo en equipo en entornos virtuales. Revista de Universidad y Sociedad del Conocimiento, 4(1). Recuperado de: http://www.raco.cat/index.php/RUSC/article/vie $\underline{\mathrm{w} / 58126 / 68218}$

Guzmán, V. \& Irigoin, M.E. (2000). Módulo de Formación para la Empleabilidad y la Ciudadanía. Documento de Base para el Diseño Curricular. Organización Internacional del Trabajo (Cinterfor/OIT), Montevideo. Recuperado de: http://ilomirror.library.cornell.edu/public/spanish/region/a mpro/cinterfor/publ/mod_form/pdf/doc._base.pdf

Gros, B.; García, I. \& Lara, P. (2009). El desarrollo de Herramientas de apoyo para el Trabajo Colaborativo en Entornos Virtuales de Aprendizaje. Revista Iberoamericana de Educación a Distancia, 12(2). doi: http://doi.org/10.5944/ried.2.12.903

Guasch, T.; Guárdia, L. \& Barberá, E. (2009). Prácticas del portafolio electrónico en el ámbito universitario del Estado Español. Revista de Educación a Distancia, Monográfico VIII. doi: http://doi.org/10.1111/j.1467-8535.2007.00803.x
Herradón, R.; Blanco, J.; Pérez, A. \& Sánchez, J.A. (2009). Experiencias y metodologías blearning para la formación y evaluación en competencias genéricas en Ingeniería. $L a$ Cuestión Universitaria, 5, 33-45. Recuperado de: http://www.lacuestionuniversitaria.upm.es/web/g rafica/articulos/imgs_boletin_5/pdfs/LCU5-4.pdf

Ibarra Sáiz, Ma.S. (dir.) (2008). Proyecto Evalcomix: Evaluación de competencias en un contexto de aprendizaje mixto (blendedlearning). Programa de estudios y análisis para la mejora de la calidad de la enseñanza superior y de la actividad del profesorado universitario. Cádiz: Servicio de Publicaciones de la Universidad de Cádiz.

Ibarra Sáiz, Mª.S. \& Rodríguez Gómez, G. (2010). Los procedimientos de evaluación como elementos de desarrollo de la función orientadora en la universidad. Revista Española de Orientación y Psicopedagogía, 21(2), 443-461. doi:

https://doi.org/10.5944/reop.vol.21.num.2.20 $\underline{10.11558}$

Iriondo, W. \& Gallego, D. (2013). El currículo y la educación a distancia. Revista Iberoamericana de Educación a Distancia, 16(1), 109-132. doi: http://doi.org/10.5944/ried.16.1.2062

Jornet, J.M.; González, J.; Suárez, J. \& Perales. $M^{\mathrm{a}}$.J. (2011). Diseño de procesos de evaluación de competencias: consideraciones acerca de los estándares en el dominio de las competencias. Bordón, 63(1), 125-145.

Lázaro y Torres, M.L.; Ruíz Palomeque, M.E. \& González González, M.J. (2009). La utilización de Hot Potatoes en el Campus Virtual. Moodle como herramienta de autoevaluación. Comunicación presentada en la V Jornada Campus Virtual UCM: Buenas prácticas e indicios de calidad, Madrid. Recuperado de: http://eprints.ucm.es/9957/1/10_lazaro.pdf

Le Boterf, G. (2001). Ingeniería de las competencias. España: gestión 2000.

Le Deist, F.D. \& Winterton, J. (2005). ¿What is competence? Human Resource Development International, $8, \quad 27-46 . \quad$ doi: https://doi.org/10.1080/1367886042000338227

Maldonado, L.F.; Leal, L.A. \& Montenegro, M. (2009). Análisis e interacciones en foro y chat: consolidación de grupo y liderazgo comunicativo en un curso de lógica matemática. Revista 
Iberoamericana de Educación a Distancia, 12(2), 189-210. doi: http://doi.org/10.5944/ried.2.12.907

Martín, A.; León, C. \& García, A. (2014). Innovación docente para la integración de Autoformación y autoevaluación en la plataforma WEBCT. Píxel-Bit. Revista de Medios y Educación, 44, 201-2014. doi: http://dx.doi.org/10.12795/pixelbit.2014.i44.14

Martín Sánchez, M.A. \& López Meneses, E. (2012). La sociedad de la información y la formación del profesorado. E-actividades y aprendizaje colaborativo. Revista Iberoamericana de Educación a Distancia, 15(1), 15-35. doi: http://doi.org/10.5944/ried.1.15.775

Martínez, J. (2008). Competencias genéricas y transversales. La opinión de los profesores universitarios. En C. Hué García (coord.), Competencias genéricas y transversales de los titulados universitarios. Recuperado de: http://www.unizar.es/ice/images/stories/publicaci onesICE/Col.\%20Documentos\%2008.pdf

McMurtrey, M. E., Downey, J. P., Zeltmann, S. M. \& Friedman, W. H. (2008). Critical skill sets of entry-level IT professionals: An empirical examination of perceptions from field personnel. Journal of Information Technology Education, 7, 101-120.

Mertens, L. (1997). Competencia Laboral: sistemas, surgimiento y modelos. Organización Internacional del Trabajo (Cinterfor/OIT), Montevideo. Recuperado de: http://www.oei.es/etp/competencia laboral siste mas modelos mertens.pdf

Moreno-Crespo, P.; López Noguero, F. \& Cruz Díaz, M. (2014). Portafolio digital: un nuevo formato de aprendizaje. Didáctica de las Ciencias Experimentales y Sociales, 28, 83-94. doi: https://doi.org/10.7203/dces.28.3182

Myers, T., Blackman, A., Andersen, T., Hay, R. \& Lee, I. (2014). Cultivating ict students' interpersonal soft skills in online learning environments using traditional active learning techniques. Journal of Learning Design, 7(3), 3853. doi: https://doi.org/10.5204/jld.v7i3.194

Nicol, D. (2009a). Assessment for learner selfregulation: enhancing achievement in the first year using learning technologies. Assessment \& Evaluation in Higher Education, 34(3), 335-352.
Nicol, D. (2009b). Transforming assessment and feedback: enhancing integration and empowerment in the first year. Quality Assurance Agency, Scotland. Recuperado de: http://www.reap.ac.uk/Portals/101/Documents/R EAP/transforming-assessment-and-feedback.pdf

Nicol, D. (2010). From monologue to dialogue: improving written feedback in mass higher education. Assessment \& Evaluation in Higher Education, 35(5), 501-517. doi: https://doi.org/10.1080/02602930802255139

Nicol, D. \& Macfarlane, D. (2006). Formative assessment and self-regulated learning: a model and seven principles of good feedback practice. Studies in Higher Education, 31(2), 199-218. Doi:

https://doi.org/10.1080/03075070600572090

Palmer, A., Montaño, J. \& Palou, M. (2009). Las Competencias Genéricas en la Educación Superior. Estudios comparativo entre la opinión de empleadores y académicos. Psicothema, 21(3), 433-438.

Olmos Migueláñez, S. \& Rodríguez Conde, M.J. (2008, 1-13 de octubre). Autoevaluación online a través de Perception como estrategia orientadora del aprendizaje de los alumnos. En A. B. Gil, J. A. Velázquez \& F. J. García (Coords.), X Simposio Internacional de Informática Educativa SIIE, 133-139, Salamanca.

Peñalosa, E. (2010). Evaluación de los aprendizajes y estudios de la interactividad en entornos en línea: un modelo para la investigación. Revista Iberoamericana de Educación a Distancia, 13(1), 17-38. doi: http://doi.org/10.5944/ried.1.13.886

Pérez, A. \& Soto, E. (2009). Competencias y Contextos Escolares: Implicaciones Mutuas. Organización y Gestión Educativa: Revista del Fórum Europeo de Administradores de la Educación, 17(2), 17-21.

Perrenoud, P. (2004). Desarrollar la práctica reflexiva en el oficio de enseñar. Barcelona: Grao.

Rodríguez, G. (dir.) (2008). Proyecto Evalhida: Evaluación de competencias con Herramientas de Interacción Dialógica Asíncronas (foros, blogs y wikis). Servicio de Publicaciones de la Universidad de Cádiz.

Rodríguez, R.; Hernández, N. \& Díaz, M.A. (2007). Cómo planificar asignaturas para el 
aprendizaje de competencias. Documentos Instituto de Ciencias de la Educación, Universidad de Oviedo.

Rogmann, J.J. (2008). Evaluating ComputerSupported Social Learning An Integrative Study of the Development of Soft Skills in a Universitybased mediation training programs. Hamburg, Germany: Universität Hamburg, Fachbereich Psychologie.Germany:

Hamburg

University..Tesis Doctoral.

Ruíz Morales, Y. (2013). Evaluación de competencias genéricas en la universidad. Estudio comparativo en entorno b-learning $y$ presencial. Madrid: UCM Tesis Doctoral.

Sáez, J. (2009). El enfoque por competencias en la formación de los educadores sociales: una mirada a su caja de herramientas. Pedagogía Social Revista Interuniversitaria, 16, 9-20. doi: https://doi.org/10.7179/PSRI_2009.16.02

Sancho Thomas, P. (2009). Un Sistema para el Aprendizaje Virtual Colaborativo Escenificado a través de un Juego de Rol Multi-Jugador. Madrid: UCM Tesis Doctoral.

Sangrá, A. (2001). Enseñar y aprender en la virtualidad. Educar, 28, 117-131.

Suárez, J.M.; Pérez, A.; Boza, A. \& GarcíaValcárcel, A. (2013). Educación 2.0. Horizontes de la innovación en la educación. En C. Jiménez; J.L. García; B. Álvarez \& J. Quintana (eds.): Investigación y educación en red, (19-60). Madrid: McGrawHill.

Tejada, J. (2005). El trabajo por competencias en el prácticum: cómo organizarlo y cómo evaluarlo. Revista Electrónica de Investigación Educativa, 7(2). DOI: http://doi.org/10.3916/C44-2015-06

Tobón, S.; Pimienta, J. \& García Fraile, J.A. (2010). Secuencias didácticas: aprendizaje y evaluación de competencias. México: Pearson.
Valverde Berrocoso, J. \& Ciudad Gómez, A. (2014). El uso de e-rúbricas para la evaluación de competencias en estudiantes universitarios. Estudio sobre fiabilidad del instrumento. REDU. Revista de docencia Universitaria, 12(1), 49-79. Recuperado de: http://redu.net/redu/index.php/REDU/article/view/724

Vargas, F. (2006). De las virtudes laborales a las competencias clave: un nuevo concepto para antiguas demandas. Boletín Cinterfor: Boletín Técnico Interamericano de Formación Profesional, 149, 13-29.

Vargas, F.; Casanova, F. \& Montanaro, L. (2001). El enfoque de competencia laboral: manual de formación. Organización Internacional del Trabajo (Cinterfor/OIT), Montevideo. Recuperado de: http://temp.oitcinterfor.org/public/spanish/region/ ampro/cinterfor/publ/man cl/index.htm

Villa, A. \& Poblete, M. (2007). Aprendizaje basado en competencias. Una propuesta para la evaluación de las competencias genéricas. Bilbao: Mensajero.

Villa, A. \& Poblete, M. (2011). Evaluación de competencias genéricas: principios, oportunidades y limitaciones. En A. De La Orden (Coord.), Educación y competencias. Bordón, 63(1), 147-170.

Zabalza, M.A. (2001). Competencias personales y profesionales en el prácticum. Resúmenes del VI Simposium Internacional sobre el Prácticum. Desarrollo de Competencias Personales y Profesiones en el Prácticum. Lugo: Unicopia. 
Ruiz-Morales, Yovanni; García-García, Mercedes; Biencinto-López, Chantal; Carpintero, Elvira (2017). Evaluación de competencias genéricas en el ámbito universitario a través de entornos virtuales: Una revisión narrativa. RELIEVE, 23(2), art. 2. doi: http://doi.org/10.7203/relieve.23.1.7183

\begin{tabular}{l} 
Authors / Autor \\
\hline Ruiz-Morales, Yovanni (yruizmorales@gmail.com) \\
Doctor en Calidad, Diversidad y Evaluación, por la Universidad Complutense de \\
Madrid. Actualmente es Profesor de metodología de la investigación en la \\
Universidad Nacional Experimental de Táchira (UNET). Es el autor de contacto \\
para este artículo. Sus principales ámbitos de investigación son el desarrollo de \\
competencias con el apoyo de las tecnologías; la e-evaluación de competencias \\
interpersonales en el contexto universitario; e-Learning y la evaluación \\
educativa. Miembro del programa de investigación en innovación y calidad \\
educativa. Su dirección postal es: Departamento de Ciencias Sociales UNET. \\
Avenida Universidad, Sector Paramillo. San Cristóbal, Venezuela. 5001
\end{tabular}

\section{García-García, Mercedes (mergaci@edu.ucm.es).}

Doctora en Ciencias de la Educación. Profesora Titular en el Departamento de Métodos de Investigación y Diagnóstico en Educación de la Universidad Complutense de Madrid (UCM). Directora del Servicio de Orientación Educativa de la Facultad de Educación. Su docencia se centra en las áreas de Pedagogía Diferencial y Orientación Educativa. Codirectora del grupo de investigación Pedagogía Adaptativa, sus líneas de investigación en los últimos años se centran en Evaluación de Estrategias de Adaptación Educativa en ESO, Tutoría universitaria en el EEES y e-Evaluación de competencias interpersonales en contextos universitarios. Su dirección postal es: Facultad de Educación UCM. C/ Rector Royo Villanova, s/n. 28040 Madrid

\section{Biencinto-López, Chantal (alameda@edu.ucm.es).}

Doctora. Es Profesora en el Departamento de Métodos de Investigación y Diagnóstico en Educación de la Facultad de Educación de la UCM. Pertenece al grupo de investigación Pedagogía Adaptativa. Entre las líneas de investigación destacan la e-evaluación de competencias interpersonales en el contexto universitario y la Metodología de Investigación. Dirección postal: Facultad de Educación UCM. C/ Rector Royo Villanova, s/n. 28040 Madrid.
To know more / Saber
más

ORCID

$\underline{0000-0003-3818-5314}$

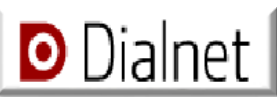

\section{Carpintero, Elvira (ecarpintero@edu.ucm.es).}

Doctora en Psicopedagogía por la UCM. Es Profesora en el Departamento de Métodos de Investigación y Diagnóstico en Educación de la Facultad de Educación. Pertenece a los grupos de investigación Pedagogía Adaptativa y Medida y Evaluación de sistemas educativos. Entre las líneas de investigación destacan la e-evaluación de competencias interpersonales en el contexto universitario y la Metodología de Investigación. Su dirección postal es: Facultad de Educación UCM. C/ Rector Royo Villanova, s/n. 28040 Madrid

\section{RELIEVE}

\section{Revista ELectrónica de Investigación y EValuación Educativa E-Journal of Educational Research, Assessment and Evaluation}

[ISSN: 1134-4032]

\footnotetext{
(C) Copyright, RELIEVE. Reproduction and distribution of this articles it is authorized if the content is no modified and their origin is indicated (RELIEVE Journal, volume, number and electronic address of the document).

(C) Copyright, RELIEVE. Se autoriza la reproducción y distribución de este artículo siempre que no se modifique el contenido y se indique su origen (RELIEVE, volumen, número y dirección electrónica del documento).
} 